\title{
Therapieziel: Selbstwirksamkeit
}

Perspektiven | Neuroreha-Tag

Neuroreha-Tag 2017 Wie Sie sich selbst und Ihre

Klienten empowern, erfahren Sie auf dem nächsten

ergotag am 29. Januar 2017. Mit Ihrer Eintrittskarte

nehmen Sie bereits einen Tag früher kostenlos am

Neuroreha-Tag teil! Dort wird es darum gehen, die

Selbstwirksamkeit von Klienten zu stärken.

\section{Veranstaltungen}

\section{ergotag, Neuroreha- Tag und TheraPro}

Am 29. Januar 2017 hören Sie auf dem ergotag in Stuttgart, wie Sie Menschen mit einer Tetraplegie dafür empowern können, ihren Alltag selbstbestimmt zu meistern. Erfahren Sie außerdem, mit welchen Mitteln Ihnen das bei Kindern, Angehörigen und Klienten mit einer psychiatrischen Erkrankung gelingt.

Mit Ihrer Eintrittskarte für den ergotag können Sie bereits einen Tag früher am 28. Januar kostenlos am Neuroreha-Tag teilnehmen und auch der Besuch der Therapiemesse TheraPro vom 27. bis 29. Januar ist inbegriffen.

Alle Infos finden Sie unter www.ergotage.de. Abonnenten von ergopraxis und neuroreha erhalten ermäßigten Eintritt.
Gerhard Schweizer hat sich nach seinem Schlaganfall zurück ins Leben gearbeitet.

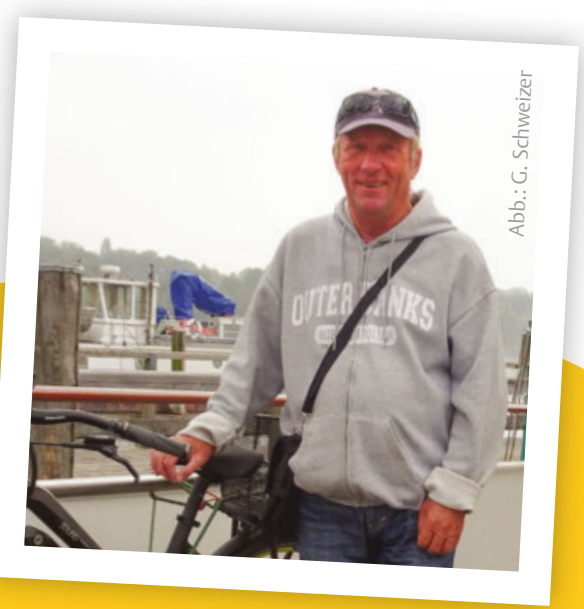

Patientenperspektive:

\section{Meine Reha - meine Ziele}

2012 erlitt Gerhard Schweizer einen Schlaganfall. Der damals 61-jährige Programmierer kehrte gerade zurück von einer Amerikareise und plante bereits die nächste, doch der Schlaganfall kam dazwischen: Hemiplegie links. Obwohl die Ärzte wenig Hoffnung hatten, lernte er wieder gehen und führt heute ein fast normales Leben. Natürlich hat sich etwas verändert, er musste sein Schiff verkaufen und sich vom Segeln verabschieden. Den Weg seiner Genesung hat er aufgeschrieben. „Schlaganfall: Das erste Jahr danach“ heißt sein Buch, mit dem er Betroffenen, Angehörigen und Freunden Mut machen möchte. Über seine Erfahrungen und warum ihm das gemeinsame Zielsetzen mit den Therapeuten wichtig war, berichtet er auf dem Neuroreha-Tag.

Außerdem erfahren Sie am 28. Januar auf dem Neuroreha-Tag:

$\rightarrow$ wie Sie mit der Zielfindung in der Neurorehabilitation zu einer verbesserten Teilhabe und zur Wiedereingliederung beitragen können.

$\rightarrow$ wie posturale Kontrolle und Selbstwirksamkeit im Alltag bei Menschen mit neurologischen Erkrankungen zusammenhängen.

$\rightarrow$ welche neuropsychologischen Hürden nach einem Schlaganfall die Wiedereingliederung erschweren.

$\rightarrow$ wie sich Schlaganfallpatienten mit ihrem betroffenen Arm alltags- und teilhabeorientierte Aufgaben/Betätigungen erarbeiten können.

$\rightarrow$ wie Sie die interprofessionelle Zusammenarbeit als Ressource einer optimierten Versorgung erkennen.

$\rightarrow$ welche Chancen die Telemedizin in der Neuroreha bietet.

Alle Infos zum Neuroreha-Tag finden Sie unter www.ergotage.de > „Neuroreha-Tag“. 